\title{
Human milk retains important immunologic properties after defatting
}

\author{
Brittany Anne Jackson, MD
}

Division of Neonatal-Perinatal Medicine, Department of Pediatrics, Michigan Medicine

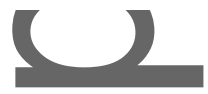

Brigid Ellen Gregg, MD

Division of Endocrinology, Department of Pediatrics, Michigan Medicine

Sara Denise Tutor, MS, RDN, CNSC, CLC

Patient Food and Nutrition Services, Michigan Medicine

Jennifer Rachelle Bermick, MD*

Division of Neonatal-Perinatal Medicine, Department of Pediatrics, Michigan Medicine Corresponding author contact information:

jendalto@med.umich.edu

1540 E. Medical Center Drive

8-621 C\&W, SPC 4254

Ann Arbor, MI 48109-4254

(734)763-4109

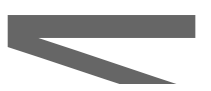

Kate Peterson Stanley, MD*

Division of Neonatal-Perinatal Medicine, Department of Pediatrics, Michigan Medicine

*These authors contributed equally to the manuscript

\section{Financial Disclosures: None declared \\ Conflicts of interest: None declared}

\begin{abstract}
Background: In neonatal chylothorax, thoracic lymphatic drainage is ineffective. The resultant effusions often require drainage, leading to a loss of immune components. Affected infants can be managed with formula or defatted human milk feedings low in long chain triglycerides to decrease lymph production. We hypothesized that there is no significant difference in the immunological profile or antibacterial effect of full fat and defatted human milk. Methods: Milk from lactating mothers was divided into one aliquot that was defatted via centrifugation with the full fat aliquot as control. Macronutrient content was analyzed with mid-infrared spectroscopy. Flow cytometry was used to measure immune cell populations. Lactoferrin, lysozyme, IgA and IgG values were determined using ELISA. The antibacterial properties were determined by inoculating paired full fat and defatted milk samples with Escherichia coli or Streptococcus pneumoniae bacteria and performing colony counts. Results: Compared to full fat milk, defatted milk demonstrated decreased total energy and fat and increased carbohydrate concentrations. Defatted milk demonstrated a significant decrease in all immune cell populations. There was no difference in IgA, IgG, lysozyme or lactoferrin concentrations. Both aliquots demonstrated equivalent
\end{abstract}

This is the author manuscript accepted for publication and has undergone full peer review but has not been through the copyediting, typesetting, pagination and proofreading process, which may lead to differences between this version and the Version of Record. Please cite this article as doi:

10.1002/jpen.1722.

This article is protected by copyright. All rights reserved. 
growth inhibition of Escherichia coli and Streptococcus pneumoniae. Conclusions: Unexpectedly, defatted human milk contained significantly less leukocytes than full fat milk. IgA, IgG, lysozyme and lactoferrin concentrations were preserved. The ability of defatted milk to inhibit bacterial growth was unaffected, suggesting that the antibacterial benefits of human milk remain after the defatting process. Further investigation regarding the clinical effect of leukocyte loss in defatted milk is warranted.

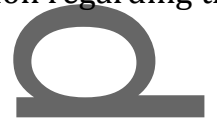

Clinical Relevancy Statement: Full fat human milk (FFHM) provides many benefits for infants, including improved neurodevelopment and decreased infection risk. The current standard of care for infants with chylothorax is feeding with a formula that is low in long chain triglycerides. Defatted human milk (DFHM) is an alternative for these infants but is labor intensive to prepare and with unclear immunologic ramifications. This study demonstrates that FFHM and DFHM contain equivalent immunologic properties, which supports the use of DFHM in infants with chylothorax.

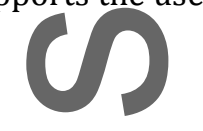

\section{Introduction:}

Neonatal chylothorax is a condition in which thoracic lymphatic drainage is ineffective, resulting in chylous pleural effusions. This can be congenital, as in lymphangiectasia, Trisomy 21 or Turner Syndrome, or acquired through surgical injury or trauma. ${ }^{1}$ These effusions can compromise cardiorespiratory function, but drainage of effusions results in a loss of immune components and can lead to a secondary immunodeficiency. ${ }^{2,3}$ Symptomatic chylothorax is initially managed by fasting, parenteral nutrition and drainage of the effusions with a thoracostomy tube. ${ }^{1,4}$ This is followed by formula feedings that are low in long chain triglycerides (LCTs), as this decreases lymph production. An alternative feeding option is defatted human milk (DFHM), which is also low in LCTs. Studies have shown that feedings with DFHM are just as effective for chylothorax resolution as feedings with low LCT formula. ${ }^{5-7}$ However, infants treated with DFHM have demonstrated reduced growth compared to those on low LCT formula in some studies, $5-7$ This discordance can be addressed by close monitoring of infant growth and customization of DFHM fortification with low LCT formula supplementation. 5-10

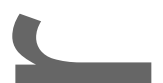

Full fat human milk (FFHM) has numerous benefits for the term and preterm infant and is the standard of care for infant nutrition..$^{11,12}$ These benefits include improved neurodevelopmental outcomes, decreased infection risk during the neonatal and infant periods, and decreased childhood obesity compared to formula fed infants. ${ }^{11,12}$ FFHM contains multiple immunologically important compounds, including maternal immunoglobulins (sIgA, sIgG), maternal immune cells (macrophages, neutrophils, lymphocytes) and anti-microbial compounds including free fatty acids, lactoferrin and lysozyme. ${ }^{13-15}$ These factors actively protect the breastfed infant from infection while simultaneously guiding neonatal and infant immune development. ${ }^{13}$ Exclusively formula fed infants do not receive these unique, individualized immunologic benefits. FFHM, which is rich in LCTs, is traditionally withheld in infants with chylothorax due to concerns that it may worsen the effusions. Because of the known benefits and immune protective properties associated with FFHM, some centers are using DFHM as the base nutrition to support infants with chylothorax rather than low LCT formula. Various methods of human milk fat removal have been devised in order to remove LCTs. ${ }^{8,9}$ The amount of fat in DFHM typically ranges from $<0.5 \mathrm{~g}$ fat $/ \mathrm{dl}$ to $0.9 \mathrm{~g}$ fat/dl depending on the study and method of measurement. 8,9 While triglyceride content has been reported as the main outcome measure when comparing defatting methods, changes in immunological properties and other macronutrients have not been widely described. ${ }^{5}$ Some immunologically important

This article is protected by copyright. All rights reserved. 
proteins, including lactadhedrin and immunoglobulins, are located within milk fat globules, so it is plausible that immune related factors could be affected by the defatting process. ${ }^{16,17}$ However, we hypothesized that there would be no significant difference in the immunological profile or antibacterial effect of full fat and defatted human milk and sought to fill this gap in the literature.

In this study, we measured macronutrient content, immune cell populations, antimicrobial proteins and bacterial growth inhibition in human milk before and after the defatting process. The DFHM samples contained less fat and total energy compared to the FFHM samples, while the remaining macronutrients were not significantly changed. The DFHM samples contained equivalent lactoferrin, lysozyme, sIgA and sIgG compared to the FFHM samples, but had decreased immune cell populations, including T cells, B cells, monocytes and neutrophils. Despite this, DFHM demonstrated equivalent growth inhibition of the bacteria Escherichia coli (E. coli) and Streptococcus pneumoniae (S. pneumoniae).

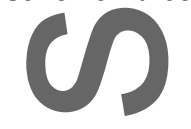

Methodology:

The University of Michigan Institutional Review Board approved this research protocol. Term (37-42 weeks' gestation) and preterm ( $<37$ weeks' gestation) infants admitted to the C.S. Mott Children's Hospital were screened to determine eligibility. Exclusion criteria included mothers $<18$ years old and mothers who were not primarily English speaking. Lactating mothers were offered enrollment into the study and written informed consent was obtained. Mothers provided a $30 \mathrm{~mL}$ morning expression of FFHM from one breast at least 2 hours after the most recent feeding. To ensure that near mature milk was obtained rather than colostrum, samples were collected between 10-60 days of lactation for term infants and between 10-28 days of lactation for preterm infants. ${ }^{18,19}$ Samples were collected, deidentified and assigned a study number. Sample sizes for individual experiments are detailed in the accompanying graphs.

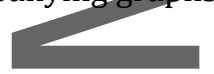

Samples were stored at $4^{\circ} \mathrm{C}$ and were processed within 4 days of expression, which is the recommended limit of storage time for fresh breast milk by the Academy of Breastfeeding Medicine. ${ }^{20}$ Each maternal sample was divided into two aliquots for evaluation and comparison. One $10 \mathrm{~mL}$ aliquot remained whole for analysis (FFHM). The remaining $20 \mathrm{~mL}$ was processed in a centrifuge $\left(2^{\circ} \mathrm{C}, 3000 \mathrm{RPM}\right.$ for 15 minutes) to separate the DFHM from the cream layer using the Hermle Z446K centrifuge. The DFHM was poured off to bypass the cream layer disk into a new bottle for testing. Creamatocrit testing on a Medela Crematocrit Plus was performed per manufacturer instructions to ensure the sample was adequately defatted with a value of $<3 \%$ cream. FFHM and DFHM samples were either used fresh or were frozen at $20^{\circ} \mathrm{C}$.

\section{Macronutrients:}

Stored frozen FFHM and DFHM samples $(5 \mathrm{~mL})$ were thawed on ice, then warmed to $40^{\circ} \mathrm{C}$ using a bead bath (Miris heater). Samples were sonicated with ultrasonic homogenization (Miris Ultrasonic Processor, $1.5 \mathrm{~s} / \mathrm{mL}$ ) immediately prior to total macronutrient analysis. FFHM and DFHM samples were analyzed for total macronutrient content using mid-infrared transmission spectroscopy MIRIS HMATM (MIRIS Human Milk Analyzer, Sweden) according to manufacturer recommendations. Samples were run in duplicate for 
fat, crude protein, true protein, carbohydrate, total solid, and energy. Average macronutrient content was used for analysis.

\section{Flow Cytometry:}

Fresh FFHM and DFHM samples were spun down at $600 \mathrm{~g}$ for 15 minutes at $4{ }^{\circ} \mathrm{C}$ to obtain a cell pellet. The cell pellet was washed twice with flow buffer (PBS with $1 \%$ fetal calf serum and $0.002 \mathrm{M}$ EDTA). Fc receptors were blocked with purified human IgG. Cells were labeled according to the manufacturer's instructions using the following antibodies (clones) from BioLegend: CD3 (HIT3a, Pacific Blue), CD14 (M5E2, Brilliant Violet 650) and CD16 (B73.1, FITZ); BD Bioscience: CD11b (ICRF44, APC); and eBioscience: CD19 (HIB19, PE) each at a 1:100 dilution. Flow cytometry was performed using a NovoCyte with 405, 488 and 640 nm lasers. Data was analyzed using FlowJo version 10.

\section{ELISA:}

Stored frozen FFHM and DFHM samples were thawed at room temperature. Samples were diluted within manufacturer recommended ranges as follows: IgA 1:20,000 dilution, IgG 1:1,000 dilution, Lysozyme 1:2,000 dilution, Lactoferrin 1:200,000 dilution. ELISA analysis was performed for each sample per Abcam protocol for human milk in the following manuals: IgA Human SimpleStep ELISA Kit ab196263 (updated Oet 20, 2015), IgG Human SimpleStep ELISA Kit ab195215 (updated Aug 14, 2015), Lysozyme Human ELISA Kit ab108877 (updated May 18, 2016) and Human Lactoferrin SimpleStep ELISA Kit ab200015 (updated Aug 17, 2017).

\section{Bacterial Growth Inhibition:}

Fresh FFHM and DFHM samples were inoculated with Escherichia coli (E. coli, ATCC 700973) or Streptococcus pneumoniae (S. pneumoniae, ATCC 6303). The OD600 of the samples was approximately 0.257 , which is equivalent to a McFarland standard 1.0, as previously published. ${ }^{21} 50$ microliters of the bacteria were added per $\mathrm{mL}$ to full fat milk, defatted milk, tryptic soy broth (E. coli) or Todd Hewitt broth with $0.1 \%$ yeast extract (S. pneumoniae). E. coli containing samples were plated on tryptic soy agar and $S$. pneumoniae containing samples on sheep's blood agar in serial dilutions. Negative controls of both FFHM and DFHM not inoculated with bacteria were plated on tryptic soy agar. Prior to plating, E. coli inoculated and negative control samples were incubated at $37^{\circ} \mathrm{C}$ with shaking at 200 RPM. S. pneumoniae samples were incubated at $37^{\circ} \mathrm{C}$ with $5 \% \mathrm{CO} 2$ without shaking. Negative controls and bacterial samples were plated at 2-hour intervals from 0 to 8 hours and incubated at $37^{\circ} \mathrm{C}$ with $5 \% \mathrm{CO}$. Bacterial colony forming units (CFU) were assessed 24 hours after plating to determine the effect of the different milks on bacterial growth. Percent growth inhibition was calculated as [(bacterial CFU in control broth - bacterial CFU in breastmilk)/bacterial CFU in control broth] x 100.

\section{Statistical Analysis:}

Parametric paired t-tests were performed on macronutrients, lactoferrin, lysozyme, sIgG and sIgA. Nonparametric Wilcoxon matched-pairs signed rank test was performed for all cell populations. Multiple paired t-tests were used for the bacterial inhibition arm of the study with statistical significance for each time point determined using the Holm-Sidak method. $p$-values of $<0.05$ were considered significant. For each DFHM sample the mother's own FFHM sample was used as its control.

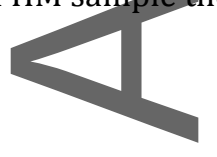

This article is protected by copyright. All rights reserved. 


\section{Results}

\section{DFHM contained significantly less fat and total energy than FFHM.}

Human milk defatting significantly decreases total fat and triglyceride content while leaving electrolyte and trace mineral content largely unchanged. 5,22 The effect of human milk defatting on other

macronutrients, including carbohydrate and protein content, remains unknown. Mid infrared spectroscopy was used to compare the macronutrient content of FFHM and DFHM from the same donor, including total energy, fat, carbohydrate and protein content. DFHM contained significantly less total energy and fat than FFHM in mothers of both term and preterm infants (Figure $1 \mathrm{~A}-\mathrm{B}, \mathrm{p}<0.01$ ). The defatting process resulted in increased total carbohydrate content in both term and preterm human milk samples (Figure 1C, p<0.01). The total protein content of DFHM remained unchanged in preterm samples, with a slight decrease noted in term samples (Figure 1D, $\mathrm{p}<0.01$ ). Overall, DFHM contained less total energy and fat with increased carbohydrate content compared to FFHM.

\section{Immune cell populations decreased during the defatting process, but other immune factors remained unchanged.}

Human milk contains numerous maternally derived immune cell populations, including monocytes, $\mathrm{T}$ cells, B cells, natural killer cells, neutrophils and eosinophils. ${ }^{13,23}$ These cells are thought to protect the mammary gland from infection, provide active immunity for the infant and guide the development of immunocompetence in the infant. ${ }^{13,23}$ It is unclear what effect the defatting process has on human milk immune cell populations. Flow cytometry was used to compare populations of total leukocytes, T cells, B cells, monocytes and neutrophils between FFHM and DFHM. DFHM contained significantly fewer total leukocytes, T cells, B cells, monocytes and neutrophils than FFHM in both term and preterm samples (Figure $2 \mathrm{~A}-\mathrm{C}_{2} \mathrm{p}<0.0001$ ). Human milk contains many anti-microbial compounds including lactoferrin, lysozyme, slgA and sIgG. Lactoferrin is one of the most abundant proteins in human milk. It binds free iron and makes it unavailable for bacterial growth and exerts iron-independent bactericidal activity. ${ }^{24}$ Lysozyme is an enzyme that degrades both gram-positive and gram-negative bacterial cell walls, serving as a natural antibiotic. ${ }^{25}$ sIgA and sIgG pass readily through the breastmilk, providing the infant with protection against pathogens the mother has encountered. ${ }^{25}$ We sought to determine if the defatting process had an impact on anti-microbial proteins found in human milk. ELISA was used to evaluate the levels of lactoferrin, lysozyme, sIgA and sIgG in FFHM and DFHM. DFHM and FFHM from preterm mothers contained equivalent amounts of lactoferrin, while DFHM from term mothers contained slightly less lactoferrin than FFHM (Figure 3A, p<0.05). There was no difference in the concentrations of lysozyme, sIgA or sIgG between DFHM and FFHM (Figure 3B-D). Overall, DFHM contained significantly fewer immune cells, but equivalent anti-microbial proteins compared to FFHM except for the decrease in lactoferrin in term DFHM samples.

\section{DFHM and FFHM demonstrated equivalent bacterial growth inhibition.}

Unpasteurized FFHM contains numerous bioactive compounds that inhibit bacterial growth. ${ }^{21,26}$ To determine the effect of defatting human milk on bacterial growth, we inoculated FFHM and DFHM with $E$. coli or S. pneumonide and measured bacterial growth inhibition. These bacteria were chosen because they are leading causes of neonatal sepsis and are some of the most likely pathogens neonates and infants might encounter while breastfeeding. ${ }^{27}$ FFHM and DFHM demonstrated equivalent bacterial growth inhibition of both E. coli and S. pneumoniae up to 8 hours after inoculation (Figure 4A-B). Although DFHM contained significantly fewer immune cells, it demonstrated equivalent anti-microbial activity compared to FFHM.

This article is protected by copyright. All rights reserved. 


\section{Discussion}

DFHM is becoming a widely-accepted alternative to low LCT formula in the treatment of neonatal chylothorax, but no studies have evaluated the global macronutrient composition and immunologic properties of DFHM. In this study, we demonstrated that DFHM contained less total energy and fat than FFHM, but that carbohydrate and protein content were equal or slightly increased. DFHM contained significantly fewer total immune cells and immune cell subpopulations than FFHM, but the anti-microbial proteins lactoferrin, lysozyme, sIgA and sIgG were conserved. Most significantly, bacterial growth inhibition was preserved in DFHM.

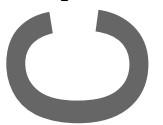

As expected, DFHM contained less total energy and fat than FFHM. ${ }^{5-7}$ Consistent with a previous report, we found that DFHM contained an increased concentration of carbohydrates, with an average increase of $0.2 \mathrm{~g} / 100 \mathrm{~mL}$ compared to FFHM. ${ }^{28}$ This increase is unlikely to be clinically relevant and is most likely due to a reduction in the total volume of liquid available for suspension after the defatting process, though this is speculation. After defatting, DFHM is deficient in not only fat, but total calories, essential fatty acids and fat-soluble vitamins. ${ }^{29}$ In practice, these deficiencies can be managed by supplementing the DFHM with low LCT formula and vitamins. ${ }^{7,9}$ It is important to note that excessive carbohydrate intake increases the respiratory quotient and stimulates lipogenesis and fat deposition, which could have long-term consequences for infant growth and metabolism. ${ }^{30,31}$ Infants who are fed DFHM may receive a slightly higher concentration of carbohydrates compared to infants fed FFHM, so they should be carefully monitored for growth. Fortification should be tailored to each individual infant's needs to optimize growth and development and to ensure they are receiving appropriate ratios of macronutrients.

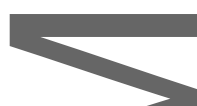

Total leukocytes and all immune subpopulations were significantly decreased in DFHM. This is likely due to the method of defatting, as it involved centrifugation followed by decanting of the non-fat containing milk. This decanting method left the immune cell pellet in the bottom of the centrifugation tube rather than in the DFHM. Although we did not evaluate for the presence of epithelial, progenitor or stem cells in DFHM, it is tikely that they were also decreased. $23,32 \mathrm{FFHM}$ is rich in leukocytes, which are believed to protect both the mammary gland and infant against infection while guiding development of the neonatal and infant immune system. ${ }^{13,14,33}$ Colostrum contains significantly more leukocytes than mature milk, but viable leukocytes have been recovered from the feces of infants fed mature human milk, suggesting that these cells remain intact and functional in the infant intestine. ${ }^{13}$ Breastmilk monocytes and macrophages perform phagocytosis, secrete immunoregulatory factors and release engulfed sIgA upon contact with bacteria in the infant intestine. ${ }^{13,34}$ Breast milk derived lymphocytes traffic from the infant's intestine to the lymph nodes, spleen and liver and are thought to promote maturation of the infant's lymphocytes. ${ }^{14}$ All of these functions are likely important for neonatal and infant immune system maturation, and it is unclear if the immune cell populations remaining in DFHM are capable of performing these functions adequately. The presence of immune cell populations in DFHM, although reduced, are likely still superior to exclusive low LCT formula feeding for neonates and infants with chylothorax. Additionally, other methods of defatting should be investigated for effectivess of retaining the full FFHM immune cell pellet. This study reinforces the need for the development of evidence based procedures for processing DFHM.

This article is protected by copyright. All rights reserved. 
Other than a slight decrease in lactoferrin in DFHM from term lactating mothers, concentrations of lactoferrin, lysozyme, sIgA and SIgG were preserved after the defatting process. Previous investigators have reported both equivalent and different concentrations of lactoferrin in breastmilk from mothers of preterm and term infants during different stages of lactation. ${ }^{35-37}$ The variability of findings within the literature may be due to the small sample sizes and different methods used to measure lactoferrin levels. ${ }^{37}$ Moreover, lactoferrin in breastmilk has also been positively associated with symptoms of infant illness and variably affected by freezing. ${ }^{38-40}$ Concentrations of lactoferrin range from $240-6501 \mu \mathrm{g} / \mathrm{mL}$ in mature preterm milk and from $240-4590 \mu \mathrm{g} / \mathrm{mL}$ in mature term milk. ${ }^{24,35-37}$ The lactoferrin concentrations in our samples fell well within these published ranges $(292-1595 \mu \mathrm{g} / \mathrm{mL}$ in preterm FFHM, 278-1854 $\mu \mathrm{g} / \mathrm{mL}$ in preterm DFHM, 355-1977 $\mu \mathrm{g} / \mathrm{mL}$ in term FFHM, 270-2111 $\mu \mathrm{g} / \mathrm{mL}$ in term DFHM). Because of this, we believe the slight decrease in lactoferrin detected in the DFHM samples from mothers of term infants is unlikely to be clinically significant and could be the result of the confounding factors noted above. Importantly, DFHM and FFHM demonstrated equivalent bacterial growth inhibition of both the common neonatal pathogens E. coli and S. pneumoniae, suggesting that the defatting process does not disrupt human milk anti-microbial function.

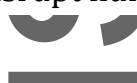

Infections caused by the loss of immunoglobulins in lymphatic fluid is a potential risk factor of congenital and post-surgical chylothorax. ${ }^{41}$ Human milk's many immunologic benefits may protect infants who have these conditions. The human milk fat globule plays a role in the infant's antimicrobial defense by releasing free fatty acids and monoglycerides via the digestive process which disrupt cell membranes of lipid coated micro-organisms including bacteria, viruses and protozoa. ${ }^{15}$ Our findings showed that the loss of milk fat in the centrifugation process did not affect the antimicrobial function of human milk against E.coliand S. pneumonia. However, Ogundele observed decreased bactericidal activity against a serum sensitive stain of E.coli in DFHM compared to FFHM. ${ }^{42}$ Further evaluation of DFHM's antimicrobial function against free fatty acid and monoglyceride targeted organisms is warranted to further understand the effects of defatting.

Our study has several limitations. First, not all assays were run on fresh milk. The macronutrient analysis and ELISA assays were run on previously frozen samples. Freezing milk has been shown to impact the concentrations of lactoferrin and IgA. ${ }^{39,40,43,44}$ However, a strength of the study is that each mother's FFHM serves as a control. As both FFHM and DFHM samples were frozen for the same length of time, we expect that any protein degradation would affect these samples equally and not significantly impact our main findings. This study was conducted at a single center, and our patient demographic is specific to our institution. Therefore, the results may not be widely generalizable. Although we assessed a range of antimicrobial proteins in this study, there are many additional immunologically important compounds present in human milk that were not included. These include cytokines, microRNAs, oligosaccharides and prebiotics, among others. $33,45,46$ While the bacterial growth inhibition of E. coli and S. pneumoniae was preserved in DFHM, the role the human milk fat globule and other immune components play in supporting infant immune function needs to be investigated. Longer term prospective studies need to be performed to-assess the impact that defatting human milk has on the risk of other infections caused by bacteria and viruses in infants with chylothorax.

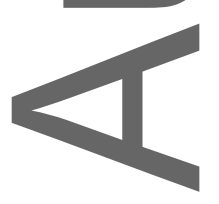

This article is protected by copyright. All rights reserved. 


\section{Conclusion}

While DFHM contained significantly fewer immune cells than FFHM, other anti-microbial properties were preserved includinglactoferrin, lysozyme, sIgA, sIgG levels and bacterial growth inhibition. Based on these results, we recommend DFHM be used as the standard of care for neonates and infants with chylothorax so they can receive the known developmental and immunologic benefits of human milk. Special care should be taken to ensure appropriate fortification of DFHM with low LCT formula to prevent growth failure in this patient population.

-

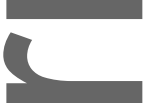

References:

1. Attar MA,Donn SM. Congenital chylothorax. Seminars in fetal \& neonatal medicine. 2017;22(4):234-239.

2. Orange/S, Geha RS, Bonilla FA. Acute chylothorax in children: selective retention of memory cells and natural killer cells. The Journal of pediatrics. 2003;143(2):243-249.

3. Hoskote AU, Ramaiah RN, Cale CM, Hartley JC, Brown KL. Role of immunoglobulin supplementation for secondary immunodeficiency associated with chylothorax after pediatric cardiothoracic surgery. Pediatr Crit Care Med. 2012;13(5):535-541.

4. Bender B, Murthy V, Chamberlain RS. The changing management of chylothorax in the modern era. European journal of cardio-thoracic surgery : official journal of the European Association for Cardio-thoracic Surgery. 2016;49(1):18-24.

5. Chan GM, Lechtenberg E. The use of fat-free human milk in infants with chylous pleural effusion. Journal of perinatology : official journal of the California Perinatal Association. 2007;27(7):434-436.

6. Fogg KL, DellaValle DM, Buckley JR, Graham EM, Zyblewski SC. Feasibility and Efficacy of Defatted Human Milk in the Treatment for Chylothorax After Cardiac Surgery in Infants. Pediatric cardiology. 2016;37(6):1072-1077.

7. Kocel SL, Russell J, O'Connor DL. Fat-Modified Breast Milk Resolves Chylous Pleural Effusion in Infants With Postsurgical Chylothorax but Is Associated With Slow Growth. JPEN J Parenter Enteral Nutr. 2016;40(4):543-551.

8. Drewniak MA, Lyon AW, Fenton TR. Evaluation of fat separation and removal methods to prepare low-fat breast milk for fat-intolerant neonates with chylothorax. Nutr Clin Pract. 2013;28(5):599-602.

9. Gilgan H, Deveau D, O'Leary G. Skimming Mother's Breast Milk at Home. ICAN: Infant, Child, \& Adolescent Nutrition. 2015;7(1):24-28.

10. Lessen R. Use of Skim Breast Milk for an Infant With Chylothorax. ICAN: Infant, Child, \& Adolescent Nutrition. 2009;1(6):303-310.

11. Breastfeeding and the Use of Human Milk. Pediatrics. 2012;129(3):e827-e841.

12. Dieterich CM, Felice JP, O'Sullivan E, Rasmussen KM. Breastfeeding and health outcomes for the mother-infant dyad. Pediatric clinics of North America. 2013;60(1):31-48.

13. Field CJ. The immunological components of human milk and their effect on immune development in infants. The Journal of nutrition. 2005;135(1):1-4.

14. Hanson LA, Korotkova M, Lundin S, et al. The transfer of immunity from mother to child. Annals of the New York Academy of Sciences. 2003;987:199-206.

15. Hamosh M, Peterson JA, Henderson TR, et al. Protective function of human milk: the milk fat globule. Seminars in perinatology. 1999;23(3):242-249.

16. Newburg DS, Peterson JA, Ruiz-Palacios GM, et al. Role of human-milk lactadherin in protection against symptomatic rotavirus infection. Lancet. 1998;351(9110):1160-1164.

This article is protected by copyright. All rights reserved. 
17. Liao Y, Alvarado R, Phinney B, Lonnerdal B. Proteomic characterization of human milk fat globule membrane proteins during a 12 month lactation period. J Proteome Res. 2011;10(8):3530-3541.

18. Koletzko B, Poindexter B, Uauy R, S. Karger (Firm). Nutritional care of preterm infants scientific basis and practical guidelines. In: Basel: Karger; 2014: http://www.karger.com/Book/Home/261508.

19. Lemons JA, Moye L, Hall D, Simmons M. Differences in the composition of preterm and term human milk during early lactation. Pediatr Res. 1982;16(2):113-117.

20. Anne-E, Lilliana S, Medicine TAoB, et al. ABM Clinical Protocol \#8: Human Milk Storage Information for Home Use for Full-Term Infants, Revised 2017. Breastfeeding Medicine. 2017;12(7):390-395.

21. Van Gysel M, Cossey V, Fieuws S, Schuermans A. Impact of pasteurization on the antibacterial properties of human milk. European journal of pediatrics.

2012;171(8):1231-1237.

22. Czank C, Simmer K, Hartmann PE. A method for standardizing the fat content of human milk for use in the neonatal intensive care unit. International breastfeeding journal. 2009;4:3.

23. Witkowska-Zimny M, Kaminska-El-Hassan E. Cells of human breast milk. Cellular \& molecular biology letters. 2017;22:11.

24. Yang Z, Jiang R, Chen Q, et al. Concentration of Lactoferrin in Human Milk and Its Variation during Lactation in Different Chinese Populations. Nutrients. 2018;10(9).

25. Lonnerdal B. Nutritional and physiologic significance of human milk proteins. The American journal of clinical nutrition. 2003;77(6):1537s-1543s.

26. Trend S, Strunk T, Hibbert J, et al. Antimicrobial protein and Peptide concentrations and activity in human breast milk consumed by preterm infants at risk of late-onset neonatal sepsis. PloS one. 2015;10(2): 0117038.

27. Camacho-Gonzalez A, Spearman PW, Stoll BJ. Neonatal infectious diseases: evaluation of neonatal sepsis. Pediatric clinics of North America. 2013;60(2):367-389.

28. Chang $\mathrm{YC}$, $\mathrm{Chen} \mathrm{CH}$, Lin MC. The macronutrients in human milk change after storage in various containers. Pediatr Neonatol. 2012;53(3):205-209.

29. Kaushik S, Wander R, Leonard S, German B, Traber MG. Removal of fat from cow's milk decreases the vitamin E contents of the resulting dairy products. Lipids. 2001;36(1):7378.

30. Minehira K, Vega N, Vidal H, Acheson K, Tappy L. Effect of carbohydrate overfeeding on whole body macronutrient metabolism and expression of lipogenic enzymes in adipose tissue of lean and overweight humans. Int J Obes Relat Metab Disord. 2004;28(10):12911298.

31. Hulst JM, van Goudoever JB, Zimmermann LJ, et al. Adequate feeding and the usefulness of the respiratory quotient in critically ill children. Nutrition. 2005;21(2):192-198.

32. Hassiotou F, Hepworth AR, Metzger P, et al. Maternal and infant infections stimulate a rapid leukocyte response in breastmilk. Clin Transl Immunology. 2013;2(4):e3.

33. Cacho NT, Lawrence RM. Innate Immunity and Breast Milk. Front Immunol. 2017;8:584.

34. Brandtzaeg P. Mucosal immunity: integration between mother and the breast-fed infant. Vaccine. 2003;21(24):3382-3388.

35. Hsu YC, Chen CH, Lin MC, Tsai CR, Liang JT, Wang TM. Changes in preterm breast milk nutrient content in the first month. Pediatr Neonatol. 2014;55(6):449-454.

36. Ronayne de Ferrer PA, Baroni A, Sambucetti ME, Lopez NE, Ceriani Cernadas JM. Lactoferrin levels in term and preterm milk. Journal of the American College of Nutrition. 2000;19(3):370-373. 
37. Villavicencio A, Rueda MS, Turin CG, Ochoa TJ. Factors affecting lactoferrin concentration in human milk: how much do we know? Biochemistry and cell biology = Biochimie et biologie cellulaire. 2017;95(1):12-21.

38. Breakey AA, Hinde K, Valeggia CR, Sinofsky A, Ellison PT. Illness in breastfeeding infants relates to concentration of lactoferrin and secretory Immunoglobulin A in mother's milk. Evolution, medicine, and public health. 2015;2015(1):21-31.

39. Rollo DE, Radmacher PG, Turcu RM, Myers SR, Adamkin DH. Stability of lactoferrin in stored human milk. Journal of perinatology : official journal of the California Perinatal Association. 2014;34(4):284-286.

40. Ahrabi AF, Handa D, Codipilly CN, et al. Effects of Extended Freezer Storage on the Integrity of Human Milk. The Journal of pediatrics. 2016;177:140-143.

41. Czobor NR, Roth G, Prodan Z, et al. Chylothorax after pediatric cardiac surgery complicates short-term but not long-term outcomes-a propensity matched analysis. Journal of thoracic disease. 2017;9(8):2466-2475.

42. Ogundele MO. Complement-mediated bactericidal activity of human milk to a serumsusceptible strain of E. coli 0111. Journal of applied microbiology. 1999;87(5):689-696.

43. Arroyo G, Ortiz Barrientos KA, Lange K, et al. Effect of the Various Steps in the Processing of Human Milk in the Concentrations of IgA, IgM, and Lactoferrin. Breastfeed Med. 2017;12(7):443-445.

44. Raoof NA, Adamkin DH, Radmacher PG, Telang S. Comparison of lactoferrin activity in fresh and stored human milk. Journal of perinatology : official journal of the California Perinatal Association. 2016;36(3):207-209.

45. Goldman AS. Future Research in the Immune System of Human Milk. The Journal of pediatrics. 2018.

46. Kosaka N, lzumi H, Sekine K, Ochiya T. microRNA as a new immune-regulatory agent in breast milk. Silence. 2010;1(1):7.

\section{Acknowledgements}

We thank Lindsay Ellsworth, MD and Emma Harman for their help with running MIRIS macronutrient analysis and drafting the methods for MIRIS analysis. We thank Lindsay Cossey and Allison Kennedy for their help with recruitment and consent.

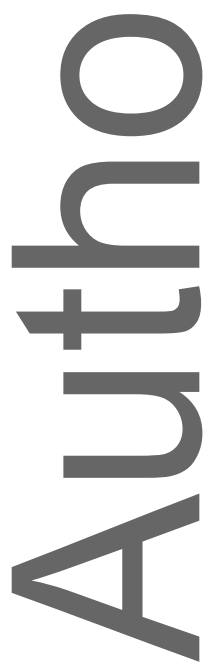

This article is protected by copyright. All rights reserved. 
Figure 1. Difference in kilocalories (A) or concentration (B-D) of energy, fat, carbohydrates and protein per $100 \mathrm{~mL}$ in full fat milk as compared to defatted milk. The boxes represent $25 \%$ ile, median and $50 \%$ ile values obtained while the whiskers represent the maximum and minimum values.

-

A: Energy

B: Fat

C: Carbohydrate

D: Protein
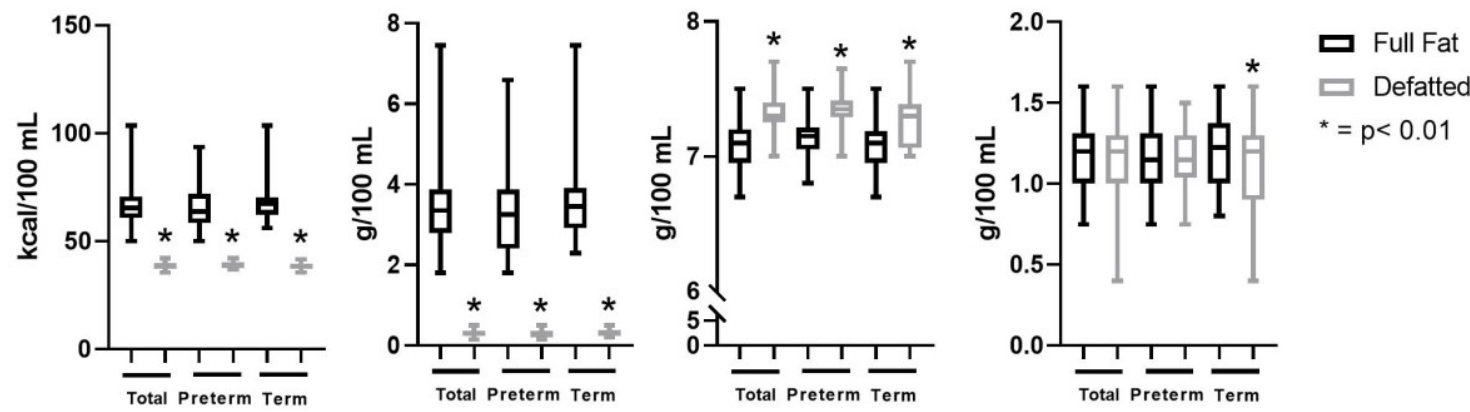

Total $n=50$ samples, Preterm $n=26$ samples, Term $n=24$ samples

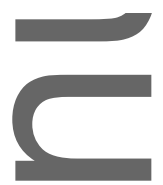

Figure 2. Differences in cells per $1 \mathrm{~mL}$ in full fat milk as compared to defatted milk. The boxes represent $25 \%$ ile, median and $50 \%$ ile values obtained while the whiskers represent the maximum and minimum values.

A: Immune Cell Subpopulations

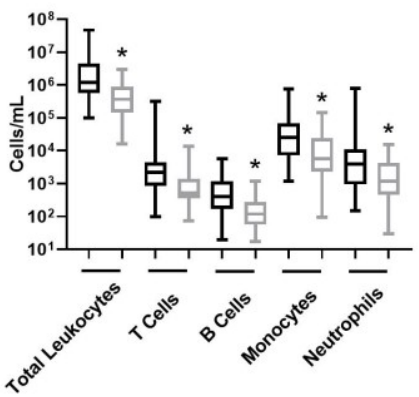

$\mathrm{n}=36$ samples

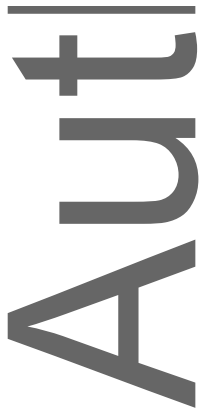

C: Term Immune Cell Subpopulations

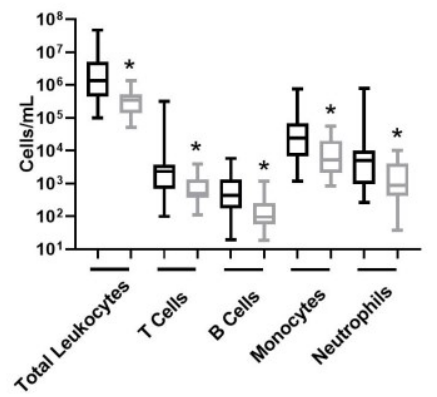

$n=19$ samples

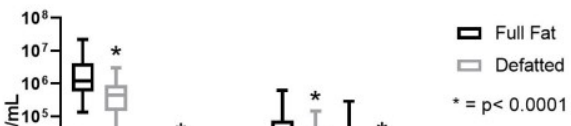

$\square$ Defatted

This article is protected by copyright. All rights reserved. 
Figure 3. Difference in concentration of immune components per $1 \mathrm{~mL}$ in full fat milk as compared to defatted milk. The boxes represent $25 \%$ ile, median and $50 \%$ ile values obtained while the whiskers represent the maximum and minimum values.
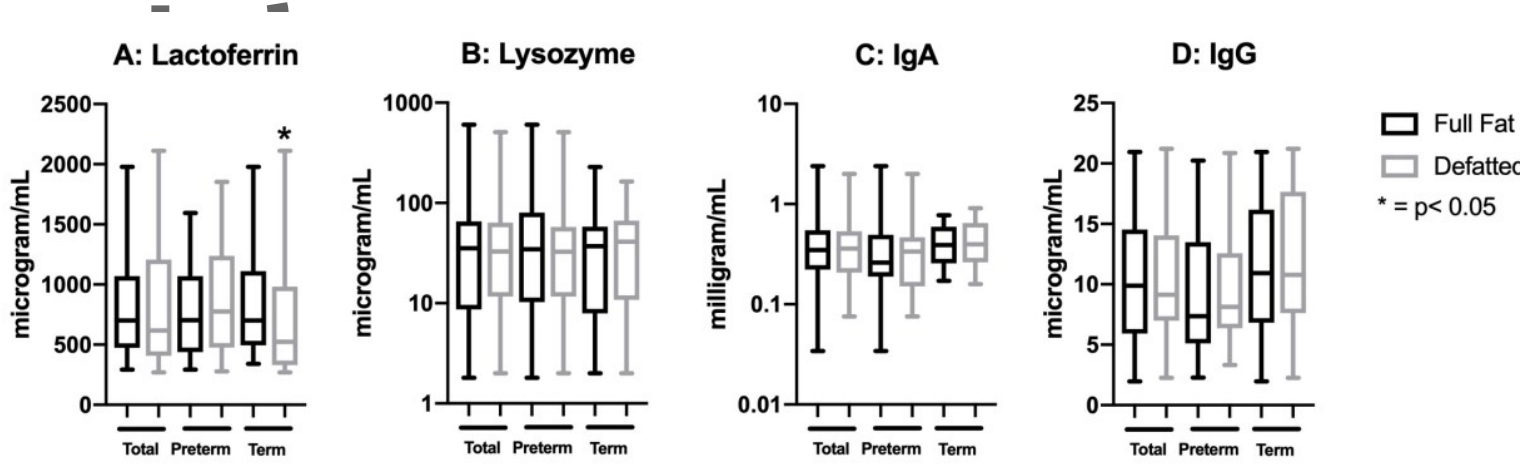

Total $n=44$ samples, Preterm $n=24$ samples, Term $n=20$ samples

Figure 4. Percent of bacterial growth inhibition in full fat and defatted milk as compared to control broth for A) Escherichia coli and B) Streptococcus pneumoniae. The points represent median growth inhibition with error bars representing $95 \%$ confidence intervals.

\section{A: Escherichia coli Growth Inhibition}

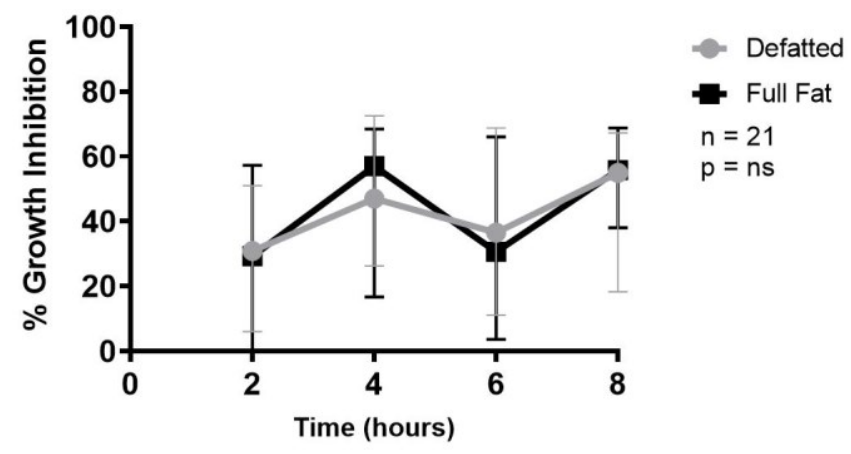

\section{B: Streptococcus pneumoniae Growth Inhibition}

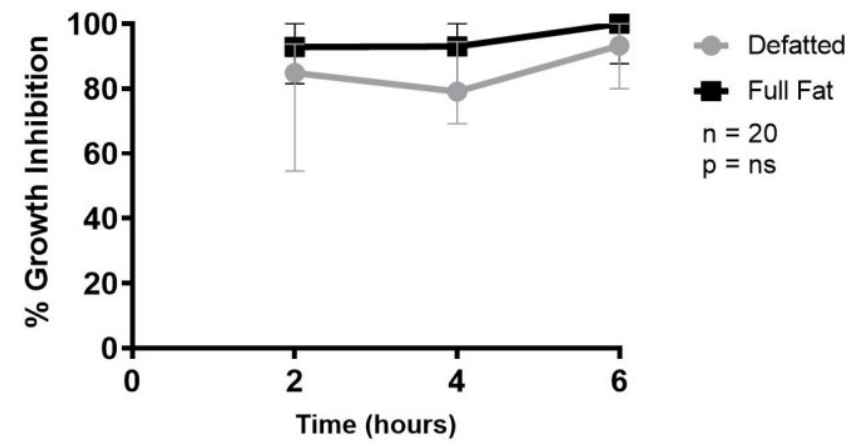

This article is protected by copyright. All rights reserved. 